\title{
REVIEW
}

Open Access

\section{The pleiotropic role of p53 in functional/ dysfunctional neurons: focus on pathogenesis and diagnosis of Alzheimer's disease}

Giulia Abate', Giovanni B. Frisoni ${ }^{2}$, Jean-Christophe Bourdon ${ }^{3}$, Simona Piccirella ${ }^{4}$, Maurizio Memo ${ }^{1}$ and Daniela Uberti ${ }^{1,5^{*}}$ (D)

\begin{abstract}
Background: Understanding the earliest pathophysiological changes of Alzheimer's disease (AD) may aid in the search for timely diagnostic biomarkers and effective disease-modifying therapies. The p53 protein is mostly known for its role in tumor suppression. However, emerging evidence supports that dysregulated p53 activity may contribute to various peripheral and brain alterations during the earliest stages of AD. This review describes the mechanisms through which p53 dysregulation may exacerbate AD pathology and how this could be used as a potential peripheral biomarker for early detection of the disease.

Main body: p53, known as the guardian of the genome, may underlie various compensation or defense mechanisms that prevent neurons from degeneration. These mechanisms include maintenance of redox homeostasis, regulation of inflammation, control of synaptic function, reduction of amyloid $\beta$ peptides, and inhibition of neuronal cell cycle re-entry. Thereby, dysregulation of p53-dependent compensation mechanisms may contribute to neuronal dysfunction, thus leading to neurodegeneration. Interestingly, a conformational misfolded variant of p53, described in the literature as unfolded p53, which has lost its canonical structure and function, was observed in peripheral cells from mild cognitive impairment (MCl) and AD patients. In AD pathology, this peculiar conformational variant was caused by post-translational modifications rather than mutations as commonly observed in cancer. Although the presence of the conformational variant of p53 in the brain has yet to be formally demonstrated, the plethora of p53-dependent compensation mechanisms underscores that the guardian of the genome may not only be lost in the periphery during AD pathology.

Conclusion: These findings revisit the role of p53 in the early development and exacerbation of AD pathology, both in the brain and periphery. The conformational variant of p53 represents a potential peripheral biomarker that could detect $A D$ at its earliest stages.
\end{abstract}

Keywords: Alzheimer's disease, p53, Conformational variant of p53

\footnotetext{
* Correspondence: daniela.uberti@unibs.it

'Department of Molecular and Translational Medicine, University of Brescia, Viale Europa, 11, 25123 Brescia, BS, Italy

${ }^{5}$ Molecular Markers Laboratory, IRCCS Istituto Centro San Giovanni di Dio Fatebenefratelli, Brescia, Italy

Full list of author information is available at the end of the article
} 


\section{Background}

Alzheimer's disease (AD) spans a clinical continuum from normal cognition to irreversible cognitive impairment, accounting for 60 to $80 \%$ of dementias [1,2]. The disease constitutes a serious health concern in the aging population with a rapidly growing socio-economic burden [3]. The worldwide economic costs associated with dementia, which consist of direct medical, direct social sector, and informal care costs, increased by 35\% between 2010 and 2015 and are estimated to rise to USD 2 trillion by 2030 [3]. A growing body of evidence demonstrates that the pathophysiological features of AD have an onset decades before the first symptoms appear $[4,5]$. During this preclinical stage, compensation or defense mechanisms, such as the DNA damage response, may initially overcome the alterations in the brain, preventing cognitive decline [6]. Some of these defense mechanisms may be dependent on p53, also known as the guardian of the genome [7]. The role of p53 beyond tumor suppression has been well established in recent years, demonstrating that dysregulated p53 signaling contributes to different pathologies including diabetes, obesity, and aging-associated neurodegenerative disorders [8]. Dependent on the stress stimulus, cell type, and context, p53 regulates the decision between life and death by either inducing apoptosis or by preventing and repairing cellular damage [8,9]. A nonsystematic review of the literature was performed, using the National Library of Medicine's PubMed database up to October 2020, to collect evidence on the possible role of p53 in the pathogenesis of AD aiming to describe how dysregulated p53 signaling may exacerbate AD pathology, while holding promise as an early predictive peripheral biomarker of the disease.

\section{AD beyond amyloid and tau pathology}

For almost 20 years, the amyloid hypothesis has been regarded as the most important driver of the pathogenesis of AD. According to this hypothesis, amyloid $\beta(A \beta)$ peptides accumulate during the process of aging forming A $\beta$ fibrils and ultimately senile plaques. This was supported by the fact that in less than $1 \%$ of cases, AD is caused by specific mutations in three genes. These genes are coding for amyloid-precursor protein (APP), Presenilin 1 (PS1), and Presenilin 2 (PS2) which are all linked to $\mathrm{A} \beta$ metabolism [10]. However, the amyloid hypothesis has come under scrutiny because immunotherapies targeting $A \beta$ failed to improve clinical outcomes in patients with mild-to-moderate AD [11-13]. It is currently unknown, if failure could be attributed to late diagnosis of patients with already severe neuronal loss or if amyloid is not the only target to fight in $\mathrm{AD}$. As demonstrated by positron emission tomography, tau accumulation is pathologically more relevant than the formation of amyloid plaques [10]. In the AD brain, tau becomes hyperphosphorylated leading to the formation of neurofibrillary tangles (NFTs). By destabilizing microtubules, these NFTs cause neuronal cell death and neurodegeneration, correlating with cognitive decline and dementia in $\mathrm{AD}$ patients [10]. However, other pathophysiological processes, including accumulation of oxidative stress, induction of a pro-inflammatory environment, and neuronal cell cycle re-entry, may precede the onset of amyloid and tau pathology.

Oxidative stress, which is the result of an imbalance between reactive oxygen species (ROS) and the activity of antioxidant enzymes, was found to be one of the earliest events contributing to synaptic impairment in $\mathrm{AD}$ [5]. $\mathrm{A} \beta$ and $\mathrm{AD}$ risk factors, such as advanced age and apolipoprotein E $\varepsilon 4$ (ApoE\&4), can all exacerbate oxidative stress [14]. Accordingly, increased markers of oxidative and nitrosative stress have been found in different post-mortem brain regions of patients with AD [15]. Furthermore, oxidative stress can induce tau hyperphosphorylation, suggesting that indeed oxidative stress is an early event in the establishment of neurodegeneration $[14,16]$.

Besides oxidative stress, the presence of immunerelated antigens and cells around amyloid plaques in the brains of patients with $\mathrm{AD}$ has been reported since the 1980s [17-19]. Both amyloid and tau pathology may be exacerbated by chronic activation of microglia, astrocytes, and other immune cells. This is further supported by emerging evidence demonstrating that mutations in microglia-specific receptors significantly increase the risk of developing AD [20]. Research is currently aiming to disentangle the exact pathophysiological mechanisms of inflammation in AD to understand how neuroinflammation may predispose patients to develop AD.

Lastly, evidence for neuronal cell cycle re-entry has been known since the mid-1990s when several phosphoepitopes characteristics of dividing cells were discovered in the AD brain but not in age-matched controls [21]. Already during the earliest stages of $\mathrm{AD}$, post-mitotic neurons re-enter the cell cycle, following either apoptosis or DNA synthesis similarly leading to cell death before cell division [22, 23]. The cell cycle hypothesis, therefore, explains the rather slow rate of atrophy during the progression of $\mathrm{AD}$. The chapters below aim to describe how dysregulation of p53 activity may underlie these different pathophysiological mechanisms and compile the evidence that p53 signaling is indeed compromised in patients progressing to AD.

\section{p53 and its isoforms: regulators of aging and neuroinflammation?}

Tight control of p53 activity is paramount for healthy aging. In fact, aberrant regulation of its activity can contribute to a broad spectrum of pathological conditions, 
spanning from accelerated aging to tumorigenesis due to excessive activation or inactivation, respectively (reviewed in $[8,24,25])$. The pleiotropic role of p53 in health and disease is dependent on the expression of at least 12 protein isoforms in addition to the expression of the full-length p53 protein [26]. Mice studies have shown that the longevityassurance activity of p53 depends on the levels of an Nterminally truncated p53 isoform $(\Delta 40 \mathrm{p} 53$ or $\mathrm{p} 44)[27,28]$. Overexpression of this isoform in mice induced accelerated aging and aggregation of tau in NFTs, which together with hyperactivation of the insulin-like growth factor signaling network caused synaptic deficits and cognitive decline, characteristic of $\mathrm{AD}[27,28]$. An additional study delineated that the $\Delta 40 \mathrm{p} 53$ isoform was generated through alternative splicing in transgenic mice overexpressing the short cytosolic APP intracellular domain (AICD) peptide, which is an important transcriptional regulator of genes involved in $\mathrm{AD}$ pathogenesis [29, 30]. Furthermore, studies have shown that this isoform is activated in an age-dependent fashion in the mouse brain coinciding with an increase in phosphorylated tau [28]. As transgenic mouse models only recapitulate parts of the pathogenesis of $\mathrm{AD}$, brain tissues of late-onset sporadic $\mathrm{AD}(\mathrm{sAD})$ patients were examined for the presence of the $\triangle 40 \mathrm{p} 53$ isoform. Levels of the $\triangle 40 \mathrm{p} 53$ isoform were only slightly but not significantly increased in these post-mortem brain tissues [29]. However, results from brain tissues may have been confounded by intrinsic subject variability and severe neurodegeneration, not completely excluding that this p53 isoform may contribute to the formation of NFTs, neurodegeneration, and accelerated aging in patients progressing to $\mathrm{AD}$ [29].

More recently, an imbalance between the expression of two different p53 isoforms was found in senescent astrocytes, which were more abundantly present in brain tissues from $\mathrm{AD}$ patients compared with controls [26]. These senescent astrocytes expressed higher levels of the senescencepromoting $\mathrm{p} 53 \beta$ isoform in comparison with the $\Delta 133 \mathrm{p} 53$ isoform, which modulates full-length p53 activity. The imbalance between these two isoforms was found to regulate the neurotoxic function of astrocytes by promoting the senescence-associated secretory phenotype which involves secretion of pro-inflammatory cytokines, such as interleukin6 [26]. Possibly, shifting the balance towards higher activity of the $\Delta 133$ p53 isoform in astrocytes could induce their neuroprotective function again, inhibiting or delaying the progression of $\mathrm{AD}$ [26]. More research will be needed to understand if an imbalance in the normal ratio of p53 and its isoforms may contribute to the development of $\mathrm{AD}$ by accelerating aging and exacerbating neuroinflammation.

\section{Dysregulation of $\mathrm{p} 53$-induced adaptive responses in the AD brain}

Besides polymorphisms and isoforms, the biological function of p53 is mainly regulated by protein-protein interactions and by a complex plethora of post-translational modifications, including phosphorylation, acetylation, and methylation [9]. These post-translational modifications subsequently orchestrate p53-induced adaptive responses, some of which may become gradually dysregulated during the continuum of $\mathrm{AD}$. Upon genotoxic stress, the stability of p53 can be increased by multiple kinases, including ataxia-telangiectasia mutated (ATM) and ATR (ATM Rad3-related) protein kinases, checkpoint kinase $1 / 2$ (Cdk1/Cdk2), and DNA-dependent protein kinase (DNAPK) $[31,32]$. Phosphorylation of p53 by one of the abovementioned kinases disrupts the p53-mouse double minute 2 (MDM2) interaction, alleviating proteasomal p53 degradation [32]. This cellular defense mechanism becomes activated under conditions of mild or subacute genotoxic stress to promote repair of DNA mutations, which naturally occur during the aging process [31]. Interestingly, microarray analysis revealed that expression of the ATM protein kinase was higher in the superior temporal cortex of $\mathrm{AD}$ patients with mild (Clinical Dementia Rating [CDR 0.5-1]) and moderate to severe (CDR 2-5) dementia compared with non-demented controls (CDR 0). Accordingly, the gene expression levels of the ATM protein kinase correlated with neuritic plaque density and Braak neuropathological stages [33]. The DNA damage response, therefore, appears to be one of the defense mechanisms that is triggered during the progression of $\mathrm{AD}$, resulting in $\mathrm{p} 53$ activation (Fig. 1).

DNA damage is often the result of oxidative stress. Under conditions of low and subacute oxidative stress, p53 ensures cell survival by activating several antioxidant genes, such as manganese superoxide dismutase (MnSOD) and TP53-induced glycolysis and apoptosis regulator (TIGAR) $[31,34]$. However, the p53-induced antioxidant response seems to be impaired in patients with AD. This finding was supported by low MnSOD expression in primary neurons isolated from an $\mathrm{AD}$ mouse model carrying mutations in APP and PS1 [35]. As MnSOD is a direct p53 target, this gradual dysregulation of the antioxidant defense during the disease continuum may be caused by impaired p 53 activity (Fig. 1).

Additionally, human studies have confirmed that p53mediated antioxidant responses are altered in postmortem brain tissues derived from patients with $\mathrm{AD}$. The metabolic pentose phosphate pathway (PPP) is known to be essential for the survival of neurons to cope with elevated production of ROS [36]. As mentioned earlier, p53 can activate TIGAR, which ensures the activity of the PPP, protecting neurons against stress-induced changes $[34,36]$. However, the expression of TIGAR was inversely correlated with the severity of $A D$ dementia [33]. Although not confirmed in human studies, dysregulation of p53 signaling in the AD brain may be responsible for the downregulation of TIGAR seen in the 


\section{Chronic exposure to mild/subacute stress during AD progression}

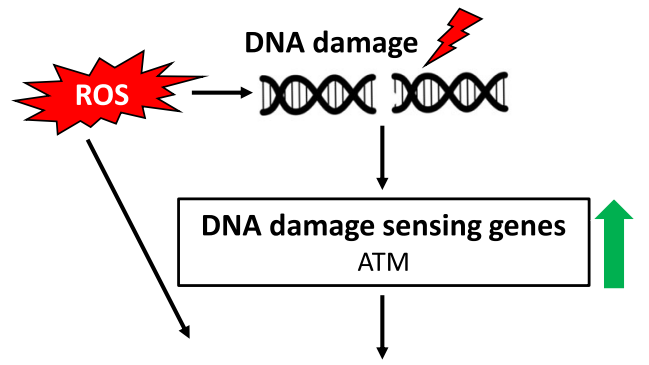

Gradual dysregulation of p53 activity

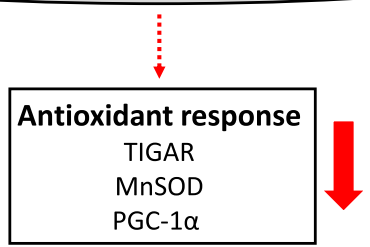

Fig. 1 Exacerbation of AD pathology through gradual dysregulation of p53-induced adaptive responses. Upon chronic exposure to mild/subacute stress, p53 becomes activated through ROS or through the induction of DNA damage sensing genes, such as ATM kinase. Following p53 activation, several antioxidant genes (e.g., TIGAR, MnSOD, and PGC-1a) are upregulated to subsequently restore cellular damage. In the AD brain, levels of ATM are strongly upregulated. However, there appears to be a defect in the downstream canonical p53 pathway, as p53-induced adaptive responses are progressively dysregulated during the continuum of the disease, exacerbating AD pathology. AD, Alzheimer's disease; ATM, ataxia-telangiectasia mutated; MnSOD, manganese superoxide dismutase; PGC-1a, peroxisome proliferator-activated receptor-c coactivator1a; TIGAR, TP53-induced glycolysis regulatory phosphatase; ROS, reactive oxygen species

superior temporal cortex of AD patients with mild (CDR 1) and severe (CDR 5) dementia (Fig. 1).

As mitochondria are the main producers of ROS, maintaining their function and viability is of the utmost importance. p53 can preserve mitochondrial function by regulating mitochondrial biogenesis, removing nonfunctional mitochondria, maintaining redox homeostasis, and controlling metabolism [36]. Dependent on the cell type and stimulus, p53 can either induce or repress peroxisome proliferator-activated receptor-c coactivator-1a (PGC-1 $\alpha$ ), which is a known inducer of mitochondrial biogenesis [36]. Interestingly, activation of PGC- $1 \alpha$ by p53 was demonstrated in neuroblastoma cells upon depletion of glutathione. Surprisingly, this activation did not increase mitochondrial biogenesis but induced an antioxidant response [37]. This p53-induced antioxidant response may also be neuroprotective in vivo, as glutathione was found to be decreased in the hippocampi of patients with mild cognitive impairment (MCI) (CDR 0.7 and Mini-Mental State Examination [MMSE]: 25.5) and AD (CDR 1.5 and MMSE: 18.4). The study confirmed that this decrease in glutathione was not attributed to tissue atrophy [38]. Furthermore, the reduced glutathione levels significantly correlated with the degree of cognitive impairment. Interestingly, also PGC- $1 \alpha$ expression was found to decrease with the severity of dementia when examining the hippocampi of AD patients with different neuritic plaque and $\mathrm{A} \beta_{\mathrm{X}-42}$ contents. These findings again imply that loss of the p53-induced adaptive response is involved in the exacerbation of oxidative stress early on during the progression of $\mathrm{AD}$ [39] (Fig. 1). As a result of increased oxidative stress, proinflammatory genes may be induced, shifting the immune balance towards a pro-inflammatory phenotype [40]. Emerging evidence suggests that also p53 itself may modulate the pro-inflammatory environment [41], but the exact implications for $\mathrm{AD}$ remain to be deciphered.

Although the above-described evidence suggests that impairment of p53-induced adaptive responses are associated with neurodegeneration observed in $\mathrm{AD}$, the link between the neuroprotective effects of p53 and AD remains controversial. This is mostly due to studies which have demonstrated that p53 is accountable for neurodegeneration due to induction of neuronal apoptosis. In vitro experiments have shown that pro-apoptotic p53 target genes are triggered upon exposure of cells to excessive levels of genotoxic/oxidative stress or micromolar $A \beta$ concentrations [42, 43]. However, neurodegeneration in $\mathrm{AD}$ is not the result of a single acute toxic insult and subsequent apoptosis, but rather due to chronic subtoxic oxidative stress insults (reviewed in [44]), resulting in the progressive loss of compensatory mechanisms, such as the p53-induced antioxidant response. Therefore, the role of p53 in AD pathology may need to be revisited, as dysregulation of p53 activity 
throughout the disease continuum may underlie the oxidative stress hypothesis, which is known to be an early determinant of the pathogenesis of $\mathrm{AD}$ [5].

\section{Revisiting the $p 53-A \beta$ feedback loop in the AD brain}

Abnormal activity of p53 may also affect other causal paths in the development of AD (Fig. 2). Wild-type p53 is a well-known inhibitor of the mammalian target of rapamycin (mTOR) signaling pathway, which is hyperactivated in the brain and periphery before the AD dementia stage [45-47]. In vitro experiments using primary neuron cultures from mice have shown that hyperactivation of the mTOR signaling axis was in part mediated by the accumulation of $A \beta$, which activates mTOR complex 1 (mTORC1) at the plasma membrane. Following activation, mTORC1 phosphorylates tau at a serine residue that is required to induce aberrant cell cycle re-entry of post-mitotic neurons [48]. Additionally, a study using an $\mathrm{AD}$ transgenic mouse model found that buildup of $\mathrm{A} \beta$ increased the activity of the mTOR pathway initiating a negative feedback loop which reduced the clearance of A $\beta$ peptides by inhibiting autophagy [49]. As mentioned earlier, mTOR signaling is also increased in human brain samples of patients affected by amnestic MCI (soluble $A \beta_{1-42}$ concentration: $29.7 \mathrm{fmol} / \mathrm{mg}$ and MMSE: 23.3) and $\mathrm{AD}$ (soluble $\mathrm{A} \beta_{1-42}$ concentration: $35.1 \mathrm{fmol} / \mathrm{mg}$ and MMSE: 12.9) in comparison with healthy controls (soluble $A \beta_{1-42}$ concentration: $8.08 \mathrm{fmol} / \mathrm{mg}$ and MMSE: 28.7) [50]. Although not formally demonstrated in AD, hyperactivation of the mTOR pathway may be caused by dysregulated p53 signaling. If unfunctional p53 is unable to repress mTOR signaling, this may lead to hyperactivation of the pathway with a concomitant inhibition of autophagy and aberrant neuronal cell cycle, linking impaired p53 signaling to both the amyloid and cell cycle hypothesis. Furthermore, in vitro experiments showed that dysregulated p53 signaling may upregulate beta-site APP-cleaving enzyme 1 (BACE1), which is the rate-limiting enzyme in the generation of $A \beta$. Interestingly, these in vitro experiments demonstrated that transcription of BACE1 is repressed by $p 53$, protecting against $A \beta$ accumulation [51]. This study, therefore, provided additional evidence that affecting the transcriptional activity of p53 can directly impact the amyloid cascade. Another p53-dependent causal path that may play a role in the development of $\mathrm{AD}$ is the downregulation of sirtuin 6 (SIRT6). SIRT6 promotes longevity by controlling DNA repair, genome integrity, energy metabolism, and inflammation [52]. Reduced levels of SIRT6 were observed in human brain samples of AD patients and the underlying mechanism was unveiled in the HT-22 mouse hippocampal neuronal cell line. In this cell line, $A \beta_{42}$ post-transcriptionally reduced $\mathrm{p} 53$ protein levels, followed by a decreased binding of p53 to the SIRT6 promotor, downregulating the expression of this anti-aging gene and subsequently compromising longevity [52].

\section{A conformational change dysregulates p53 signaling in AD}

The above-described evidence is only speculating which downstream p53 effectors could contribute to the progression of AD upon dysregulation of p53 activity. The first evidence to prove that the activity of p53 is indeed

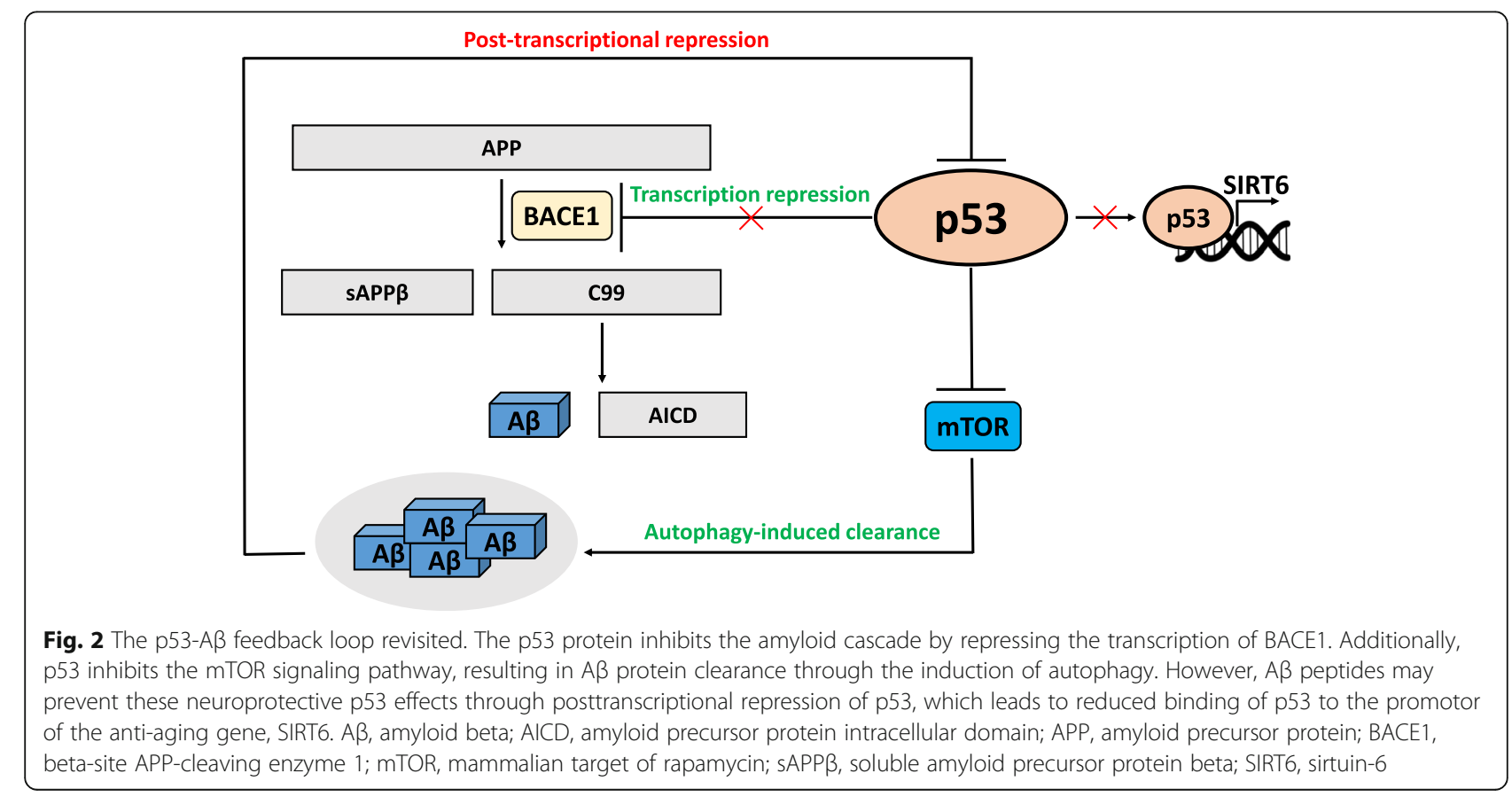


altered in $\mathrm{AD}$ was found in skin fibroblasts derived from sAD patients [53]. Upon $\mathrm{H}_{2} \mathrm{O}_{2}$ exposure, fibroblasts from sAD patients did not activate canonical p53dependent cell cycle regulator ( $p 21$ and GADD45) and pro-apoptotic $(B A X 1)$ genes. This impairment resulted in accelerated cell cycle re-entry, leading to diminished $\mathrm{H}_{2} \mathrm{O}_{2}$-induced apoptosis in fibroblasts from sAD patients compared with those from non-AD controls [53]. Although these findings were not confirmed in neurons, they were the first to demonstrate that p53-induced DNA damage repair mechanisms are altered in peripheral cells from AD patients. Lack of p53 activation was caused by a conformational change in its tertiary structure, misfolding the protein and generating a conformational variant of the p53 protein, which is transcriptionally inactive on $p 21$ and GADD45 promoters [54]. However, the conformational change was not related to mutations, as commonly is the case in tumor cells [55]. Interestingly, the mutation-independent formation of the conformational variant of the $\mathrm{p} 53$ protein was $\mathrm{AD}$-specific, as in vitro models (human embryonic kidney [HEK] and differentiated neuroblastoma SHSY5Y cells) overexpressing the $\mathrm{APP}_{751}$ protein and exposure of fibroblasts from non-AD subjects to nanomolar $A \beta_{1-40}$ and $A \beta_{1-42}$ concentrations induced the conformational change in the p53 protein (Fig. 3) [5658]. Mechanistically, low intracellular amounts of soluble $\mathrm{A} \beta$ peptides were shown to deregulate zyxin, which in turn leads to proteasomal degradation of homeodomain interacting protein kinase 2 (HIPK2) [57, 58]. Consequently, downregulation of HIPK2 resulted in the upregulation of metallothionein 2A (MT2A), inducing the conformational variant of $\mathrm{p} 53$, which is described in the literature as unfolded p53, by chelating the zinc atom that is responsible for the wild-type p53 conformation [57]. Furthermore, pretreatment of HEK cells with an A $\beta$-sequestering antibody or an antioxidant partially prevented the p53 conformational change, implying important roles of both $A \beta$ peptides and the pro-oxidant environment in p53 misfolding [56]. The pro-oxidant environment in immortalized lymphocytes derived from early-onset $\mathrm{SAD}$ and familial $\mathrm{AD}$ (fAD) patients was shown to induce the p53 conformational change, mainly due to nitration of its tyrosine residues (Fig. 3) [59]. Notably, the increase in unfolded p53 was a marker of impaired redox homeostasis due to its inverse correlation with SOD activity, considering peripheral cells from amnestic MCI (CDR 0.5), severe AD (CDR 2), and healthy subjects (CDR 0) [59, 60]. Concomitant with the increase in unfolded p53, the expression of CD44 was increased in lymphocytes from overt $\mathrm{AD}$ patients, which may be of relevance for the immune crosstalk between the periphery and brain in $\mathrm{AD}$ pathology [61]. Furthermore, a conformational change in p53 rendered lymphocytes from $\mathrm{AD}$ patients less sensitive to rapamycin-mediated inhibition of mTOR signaling, resulting in G (1)/S checkpoint

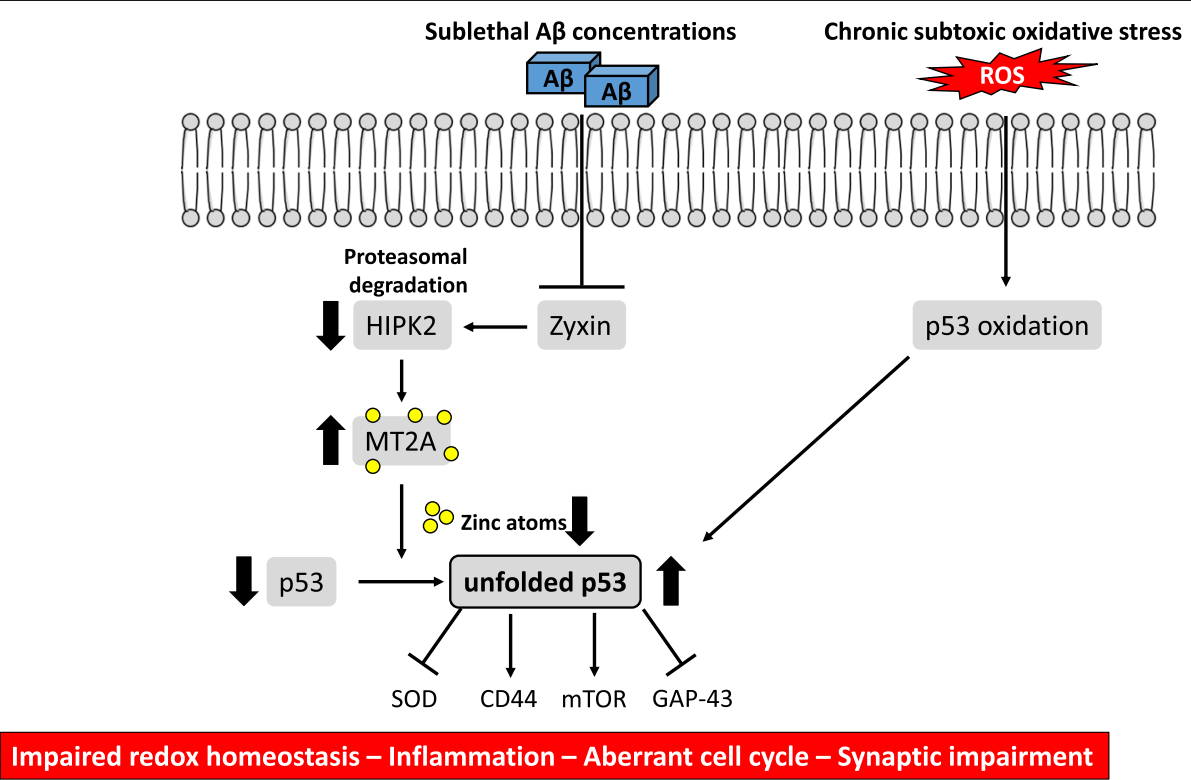

Fig. 3 The unfolded p53 protein underlies various early hallmarks of AD pathology. Sublethal A $\beta$ concentrations inhibit the Zyxin/HIPK2 signaling axis, resulting in upregulation of MT2A. Following MT2A upregulation, zinc atoms are chelated which are necessary to maintain wild-type p53 conformation. In addition, chronic exposure to subtoxic nitrosative stress results in nitration of p53 tyrosine residues, misfolding the p53 protein, and dysregulating its activity. An increase in unfolded p53 protein was directly linked to downregulation of SOD and GAP-43, and to upregulation of CD44 and mTOR, potentially leading to impaired redox homeostasis, synaptic impairment, inflammation, and aberrant cell cycle, respectively. $A \beta$, amyloid beta; GAP-43, growth-associated protein 43; HIPK2, homeodomain interacting protein kinase 2; MT2A, metallothionein 2A; mTOR, mammalian target of rapamycin; ROS, reactive oxygen species; SOD, superoxide dismutase 
dysfunction [62]. This checkpoint dysfunction could reflect aberrant neuronal cell cycle re-entry and supports the above-described hypothesis that abnormal p53 activity could contribute to dysregulated mTOR signaling in the AD brain.

Although evidence remains scarce, some studies indicate that the conformation of p53 is not only perturbed in peripheral cells but also in the brain regions of $\mathrm{AD}$ patients. An experimental study, using neuroblastoma differentiated neuron-like cells overexpressing APP, showed that unfolded p53 downregulated growth-associated protein 43 (GAP-43), which is involved in axonal outgrowth and synaptic plasticity $[63,64]$. It is indeed reported in post-mortem brain studies that GAP-43 protein expression is decreased in the frontal cortex of patients with $\mathrm{AD}[65,66]$. Although these studies did not investigate the p53 pathway, we can speculate that lack of p53 transcriptional activity, due to its conformational changes, is responsible for reduced GAP-43 expression in the AD brain. Additional evidence in post-mortem brain tissues of AD patients showed a huge amount of nitrated and oxidized p53 [67], supporting the findings in immortalized lymphocytes from AD patients. The most compelling evidence for a perturbation of p53 activity in the AD brain was recently demonstrated by Farmer et al. (2020) [68]. This study found that cytosolic p53 aggregates colocalized with tau protein in the frontal cortex of $\mathrm{AD}$ patients (Braak stage 6) but not in agematched controls [68]. It will be interesting to determine in future studies if these p53 aggregates are composed of unfolded p53. Unfolded p53 found in peripheral cells may, therefore, be resembling loss of p53 activity in the $\mathrm{AD}$ brain. If this would be the case, unfolded p53 could be a marker of various early AD pathophysiological mechanisms, such as impaired redox homeostasis, neuronal cell cycle re-entry, inflammation, and synaptic deficits (Fig. 3).

\section{Future perspectives \\ Unfolded p53: a putative peripheral biomarker for early detection of $A D$}

The assessment of biomarkers in peripheral cells and biofluids, such as fibroblasts, lymphocytes, and plasma, offers the advantage to be non-invasive and easily accessible compared to cerebrospinal fluid and imaging biomarkers. A recent review identified that $34 \%$ of reports on blood-based biomarkers were related to peripheral changes in emerging mechanisms, such as mitochondrial respiration, inflammatory markers, and oxidative stress response [69]. As described above, the conformational change in p53 may underlie these mechanisms, which may occur early in the disease continuum, supporting the use of unfolded p53 as a putative biomarker for the diagnosis of $\mathrm{AD}$ before the onset of cognitive decline. Indeed, initial studies found that the unfolded p53 protein was able to discriminate AD patients from non-AD controls, due to a significantly higher expression of this conformationally altered isoform in peripheral blood mononuclear cells (PBMCs) from overt $\mathrm{AD}$ patients (CDR 1.5-2 and MMSE: 17) [70-72]. In addition, the level of unfolded p53 was independent of the APOE $\varepsilon 4$ allele carrier status and was also significantly increased in PBMCs derived from preclinical patients with MCI compared with healthy controls. Consequently, elevated levels of the unfolded p53 protein were found to be predictive of the conversion from amnestic MCI to AD dementia [71-73]. Importantly, the unfolded p53 protein was differentially expressed between AD patients and patients affected by either Parkinson's disease or other types of dementia, confirming that the conformational change in p53 is induced by an AD-specific mechanism [70]. What still limits the general acceptance of p53 involvement in AD is the undeniable reputation it has in cancer. To overcome such view, a new antibody 2D3A8 able to identify a p53 misfolded conformational variant that was found to be highly expressed in AD has recently been developed [74]. This novel 2D3A8 antibody recognizes a linear epitope between the DNA binding domain and the conjunction region with the tetramerization domain. This epitope is less accessible when the protein is assembled in its native form but most likely becomes exposed due to redox post-translation modifications, as suggested by the pro-oxidant environment in $\mathrm{AD}$ [74]. In addition, preliminary studies found that the level of unfolded p53 protein detected by the 2D3A8 antibody in PBMCs correlated with age and cognitive impairment in $\mathrm{AD}$ [74]. Future longitudinal studies should aim to address whether this peripheral biomarker correlates with the deposition of amyloid and tau in the AD brain and confirm if unfolded p53 is able to diagnose AD at its early stages, predicting if patients with $\mathrm{MCI}$ are predisposed to develop AD.

\section{Unfolded p53: a potential target for therapeutic monitoring in $A D$}

Considering the oxidative stress hypothesis as one of the earliest mechanisms underlying $\mathrm{AD}$, antioxidant therapies have been studied for years. However, randomized clinical trials examining the clinical benefits of antioxidants, such as vitamin E supplementation, remain inconclusive. Despite the fact that vitamin E is significantly lower in plasma and cerebrospinal fluid of patients with $\mathrm{AD}$ compared to those without, vitamin E supplementation did not consistently halt cognitive decline in human trials. One of the caveats of antioxidant supplementation is the identification of appropriate oxidative stress 
markers for evaluation of its response [75]. As the unfolded p53 conformation is induced by the pro-oxidant environment [59], it has the potential to be one of these oxidative stress markers that can be used for therapeutic monitoring in studies evaluating the impact of antioxidant therapies on the disease progression of $\mathrm{AD}$. In fact, in vitro studies have shown that the formation of the unfolded p53 conformation is a dynamic process that is reversible by restoring the pro-oxidant environment [56]. Therefore, it would be interesting to examine how disease-modifying drugs may influence the conformation of p53 in the periphery and ultimately the brain of $A D$ patients. Additionally, the unfolded p53 conformation was reverted into its wild-type state upon supplementation of zinc to AD fibroblasts [57]. Although rescue of p53 conformational changes upon pharmacological and/ or immunological treatment has not been demonstrated in $\mathrm{AD}$, zinc supplementation can reactivate misfolded p53 in cancer, making it a potential cancer therapeutic [76]. Similarly, future studies may investigate whether restoration of p53 conformation could overcome the dysregulated p53 signaling in the brain potentially preventing or delaying $\mathrm{AD}$ progression.

\section{Conclusions}

The balance between the harmful and guarding effects of p53 is disturbed in various pathologies, including AD where conformational changes misfold the p53 protein altering its activity. Although the ramifications of p53 activities in the brain remain to be deciphered, emerging evidence suggests that dysregulation of its activity may contribute to various aspects of the pathogenesis of $\mathrm{AD}$. It is becoming well-established that $\mathrm{AD}$ manifests beyond the brain, with alterations in the periphery that could aid in the search for biomarkers of the disease. The conformational variant of the p53 protein, known as unfolded p53, could be one of these peripheral biomarkers, which level was significantly higher in peripheral cells derived from patients with AD compared with non-demented controls, Parkinson's disease, and other types of dementia. In addition, misfolding of the p53 protein was found to expose an $\mathrm{AD}$-specific epitope early on in the disease continuum, holding potential to predict the conversion from $\mathrm{MCI}$ to $\mathrm{AD}$. In summary, a conformational change in the p53 protein may contribute to $\mathrm{AD}$ exacerbation but holds promise as a potential peripheral biomarker for early detection of $\mathrm{AD}$.

\footnotetext{
Abbreviations

A $\beta$ : Amyloid beta; AD: Alzheimer's disease; AICD: Amyloid precursor protein intracellular domain; APOE ع4: Apolipoprotein E ع4 allele; APP: Amyloid precursor protein; ATM: Ataxia-telangiectasia mutated; ATR: ATM Rad3related; BACE1: Beta-site APP-cleaving enzyme 1; Cdk1/2: Checkpoint kinase 1/2; CDR: Clinical Dementia Rating; DNA-PK: DNA protein kinase; GADD45: Growth arrest and DNA damage-inducible 45; fAD: Familial Alzheimer's disease; GAP-43: Growth-associated protein 43;
}

HIPK2: Homeodomain interacting protein kinase 2; MCl: Mild cognitive impairment; MDM2: Mouse double minute 2; MMSE: Mini-Mental State Examination; MnSOD: Manganese superoxide dismutase; MT2A: Metallothionein 2A; mTOR: Mammalian target of rapamycin; NFTs: Neurofibrillary tangles; PBMC: Peripheral blood mononuclear cells; PGC1a: Peroxisome proliferator-activated receptor-c coactivator-1a; PPP: Pentose phosphate pathway; PS: Presenilin; ROS: Reactive oxygen species;

SAPP $\beta$ : Soluble amyloid precursor protein beta; sAD: Sporadic Alzheimer's disease; SIRT6: Sirtuin-6; SOD: Superoxide dismutase; TIGAR: TP53-induced glycolysis regulatory phosphatase

\section{Acknowledgements}

The authors would like to thank Prof. Simone Di Giovanni for critical review of the manuscript. The authors are grateful to Ismar Healthcare NV who provided medical writing assistance on behalf of Diadem srl, Brescia, Italy.

\section{Authors' contributions}

All authors contributed equally to the manuscript. All authors read and approved the final manuscript.

Funding

This study was funded by Diadem srl, Brescia, Italy.

\section{Availability of data and materials}

Not applicable.

Ethics approval and consent to participate

Not applicable.

\section{Consent for publication}

Not applicable.

\section{Competing interests}

MM is co-founder and CSO of Diadem srl; SP is an employee of Diadem srl; DU is co-founder and CSO of Diadem srl, Spin Off of Brescia University, Brescia, Italy. The ownership of the 2D3A8 antibody patent rights belongs to Diadem srl, Brescia, Italy.

\section{Author details}

${ }^{1}$ Department of Molecular and Translational Medicine, University of Brescia, Viale Europa, 11, 25123 Brescia, BS, Italy. ${ }^{2}$ Memory Clinic, University Hospitals and University of Geneva, Geneva, Switzerland. ${ }^{3}$ School of Medicine, University of Dundee, Dundee DD1 9SY, UK. ${ }^{4}$ Diadem srl, Brescia, Italy. ${ }^{5}$ Molecular Markers Laboratory, IRCCS Istituto Centro San Giovanni di Dio Fatebenefratelli, Brescia, Italy.

Received: 17 June 2020 Accepted: 23 November 2020

Published online: 03 December 2020

\section{References}

1. Aisen PS, Cummings J, Jack CR Jr, Morris JC, Sperling R, Frolich L, et al. On the path to 2025: understanding the Alzheimer's disease continuum. Alzheimers Res Ther. 2017:9(1):60.

2. Alzheimer's Association. 2019 Alzheimer's disease facts and figures. Alzheimers Dement. 2019:15(3):321-87.

3. Wimo A, Guerchet M, Ali GC, Wu YT, Prina AM, Winblad B, et al. The worldwide costs of dementia 2015 and comparisons with 2010. Alzheimers Dement. 2017;13(1):1-7.

4. Stefanova NA, Muraleva NA, Korbolina EE, Kiseleva E, Maksimova KY, Kolosova NG. Amyloid accumulation is a late event in sporadic Alzheimer's disease-like pathology in nontransgenic rats. Oncotarget. 2015;6(3):1396-413.

5. Tonnies E, Trushina E. Oxidative stress, synaptic dysfunction, and Alzheimer's disease. J Alzheimers Dis. 2017:57(4):1105-21.

6. Silva AR, Santos AC, Farfel JM, Grinberg LT, Ferretti RE, Campos AH, et al. Repair of oxidative DNA damage, cell-cycle regulation and neuronal death may influence the clinical manifestation of Alzheimer's disease. PLoS One. 2014;9(6):e99897.

7. Lane DP. Cancer. p53, guardian of the genome. Nature. 1992;358(6381): $15-6$.

8. Labuschagne CF, Zani F, Vousden KH. Control of metabolism by p53 Cancer and beyond. Biochim Biophys Acta Rev Cancer. 2018;1870(1):32-42. 
9. Vousden KH, Lane DP. p53 in health and disease. Nat Rev Mol Cell Biol. 2007;8(4):275-83.

10. Kametani F, Hasegawa M. Reconsideration of amyloid hypothesis and tau hypothesis in Alzheimer's disease. Front Neurosci. 2018;12:25.

11. Buttini M, Masliah E, Barbour R, Grajeda H, Motter R, Johnson-Wood K, et al. Beta-amyloid immunotherapy prevents synaptic degeneration in a mouse model of Alzheimer's disease. J Neurosci. 2005;25(40):9096-101.

12. Doody RS, Thomas RG, Farlow M, Iwatsubo T, Vellas B, Joffe $\mathrm{S}$, et al. Phase 3 trials of solanezumab for mild-to-moderate Alzheimer's disease. N Engl J Med. 2014;370(4):311-21.

13. Salloway S, Sperling R, Fox NC, Blennow K, Klunk W, Raskind M, et al. Two phase 3 trials of bapineuzumab in mild-to-moderate Alzheimer's disease. $N$ Engl J Med. 2014;370(4):322-33.

14. Liu Z, Li T, Li P, Wei N, Zhao Z, Liang H, et al. The ambiguous relationship of oxidative stress, tau hyperphosphorylation, and autophagy dysfunction in Alzheimer's disease. Oxidative Med Cell Longev. 2015;2015:352723.

15. Ansari MA, Scheff SW. Oxidative stress in the progression of Alzheimer disease in the frontal cortex. J Neuropathol Exp Neurol. 2010;69(2):155-67.

16. Zahid S, Khan R, Oellerich M, Ahmed N, Asif AR. Differential S-nitrosylation of proteins in Alzheimer's disease. Neuroscience. 2014;256:126-36.

17. Griffin WS, Stanley LC, Ling C, White L, MacLeod V, Perrot LJ, et al. Brain interleukin 1 and S-100 immunoreactivity are elevated in Down syndrome and Alzheimer disease. Proc Natl Acad Sci U S A. 1989;86(19):7611-5.

18. Rozemuller JM, Eikelenboom P, Stam FC. Role of microglia in plaque formation in senile dementia of the Alzheimer type. An immunohistochemical study. Virchows Arch B Cell Pathol Incl Mol Pathol. 1986;51(3):247-54.

19. Rogers J, Luber-Narod J, Styren SD, Civin WH. Expression of immune system-associated antigens by cells of the human central nervous system: relationship to the pathology of Alzheimer's disease. Neurobiol Aging. 1988; 9:339-49.

20. Kinney JW, Bemiller SM, Murtishaw AS, Leisgang AM, Salazar AM, Lamb BT. Inflammation as a central mechanism in Alzheimer's disease. Alzheimers Dement (N Y). 2018:4:575-90.

21. Vincent I, Rosado M, Davies P. Mitotic mechanisms in Alzheimer's disease? J Cell Biol. 1996;132(3):413-25.

22. Barrio-Alonso E, Hernandez-Vivanco A, Walton CC, Perea G, Frade JM. Cell cycle reentry triggers hyperploidization and synaptic dysfunction followed by delayed cell death in differentiated cortical neurons. Sci Rep. 2018;8(1):14316.

23. Yang Y, Mufson EJ, Herrup K. Neuronal cell death is preceded by cell cycle events at all stages of Alzheimer's disease. J Neurosci. 2003;23(7):2557-63.

24. Wu D, Prives C. Relevance of the p53-MDM2 axis to aging. Cell Death Differ. 2018;25(1):169-79.

25. Feng $Z$, Lin $M, W u R$. The regulation of aging and longevity: a new and complex role of p53. Genes Cancer. 2011;2(4):443-52.

26. Turnquist C, Horikawa I, Foran E, Major EO, Vojtesek B, Lane DP, et al. p53 isoforms regulate astrocyte-mediated neuroprotection and neurodegeneration. Cell Death Differ. 2016;23(9):1515-28.

27. Pehar M, O'Riordan K, Burns-Cusato M, Andrzejewski ME, del Alcazar CG, Burger $\mathrm{C}$, et al. Altered longevity-assurance activity of p53:p44 in the mouse causes memory loss, neurodegeneration and premature death. Aging Cell. 2010;9(2):174-90.

28. Pehar M, Ko MH, Li M, Scrable H, Puglielli L. P44, the 'longevity-assurance' isoform of P53, regulates tau phosphorylation and is activated in an agedependent fashion. Aging Cell. 2014;13(3):449-56.

29. Li M, Pehar M, Liu Y, Bhattacharyya A, Zhang SC, O'Riordan K, et al. The amyloid precursor protein (APP) intracellular domain regulates translation of p44, a short isoform of p53, through an IRES-dependent mechanism. Neurobiol Aging. 2015;36(10):2725-36.

30. Branca C, Sarnico I, Ruotolo R, Lanzillotta A, Viscomi AR, Benarese M, et al. Pharmacological targeting of the beta-amyloid precursor protein intracellular domain. Sci Rep. 2014;4:4618.

31. Budanov AV. The role of tumor suppressor p53 in the antioxidant defense and metabolism. Subcell Biochem. 2014;85:337-58.

32. Shieh SY, Ikeda M, Taya Y, Prives C. DNA damage-induced phosphorylation of p53 alleviates inhibition by MDM2. Cell. 1997;91(3):325-34.

33. Katsel P, Tan W, Fam P, Purohit DP, Haroutunian V. Cell cycle checkpoint abnormalities during dementia: a plausible association with the loss of protection against oxidative stress in Alzheimer's disease [corrected]. PLoS One. 2013;8(7):e68361.
34. Sablina AA, Budanov AV, Ilyinskaya GV, Agapova LS, Kravchenko JE, Chumakov PM. The antioxidant function of the p53 tumor suppressor. Nat Med. 2005;11(12):1306-13.

35. Sompol P, Ittarat W, Tangpong J, Chen Y, Doubinskaia I, Batinic-Haberle I, et al. A neuronal model of Alzheimer's disease: an insight into the mechanisms of oxidative stress-mediated mitochondrial injury. Neuroscience. 2008;153(1):120-30.

36. Wang DB, Kinoshita C, Kinoshita Y, Morrison RS. p53 and mitochondrial function in neurons. Biochim Biophys Acta. 2014;1842(8):1186-97.

37. Aquilano K, Baldelli S, Pagliei B, Cannata SM, Rotilio G, Ciriolo MR. p53 orchestrates the PGC-1alpha-mediated antioxidant response upon mild redox and metabolic imbalance. Antioxid Redox Signal. 2013;18(4):386-99.

38. Mandal PK, Saharan S, Tripathi M, Murari G. Brain glutathione levels--a novel biomarker for mild cognitive impairment and Alzheimer's disease. Biol Psychiatry. 2015;78(10):702-10.

39. Qin W, Haroutunian V, Katsel P, Cardozo CP, Ho L, Buxbaum JD, et al. PGC1alpha expression decreases in the Alzheimer disease brain as a function of dementia. Arch Neurol. 2009;66(3):352-61.

40. Chatterjee S. Chapter two - oxidative stress, inflammation, and disease. Oxid Stress Biomater. 2016:35-58. https://doi.org/10.1016/B978-0-12-803269-5. 00002-4.

41. Lowe J, Shatz M, Resnick MA, Menendez D. Modulation of immune responses by the tumor suppressor p53. BioDiscovery. 2013;8:2.

42. Lee JH, Kim HS, Lee SJ, Kim KT. Stabilization and activation of p53 induced by Cdk5 contributes to neuronal cell death. J Cell Sci. 2007;120(Pt 13):2259-71.

43. Lapresa R, Agulla J, Sanchez-Moran I, Zamarreno R, Prieto E, Bolanos JP, et al. Amyloid-ss promotes neurotoxicity by Cdk5-induced p53 stabilization. Neuropharmacology. 2019;146:19-27.

44. Lanni C, Racchi M, Memo M, Govoni S, Uberti D. p53 at the crossroads between cancer and neurodegeneration. Free Radic Biol Med. 2012;52(9): 1727-33.

45. Li X, Alafuzoff I, Soininen H, Winblad B, Pei JJ. Levels of mTOR and its downstream targets $4 \mathrm{E}-\mathrm{BP} 1, \mathrm{eEF} 2$, and $\mathrm{eEF} 2$ kinase in relationships with tau in Alzheimer's disease brain. FEBS J. 2005;272(16):4211-20.

46. Feng Z, Zhang $H$, Levine AJ, Jin S. The coordinate regulation of the p53 and mTOR pathways in cells. Proc Natl Acad Sci U S A. 2005;102(23):8204-9.

47. Yates SC, Zafar A, Hubbard P, Nagy S, Durant S, Bicknell R, et al. Dysfunction of the mTOR pathway is a risk factor for Alzheimer's disease. Acta Neuropathol Commun. 2013;1:3.

48. Norambuena A, Wallrabe H, McMahon L, Silva A, Swanson E, Khan SS, et al. mTOR and neuronal cell cycle reentry: how impaired brain insulin signaling promotes Alzheimer's disease. Alzheimers Dement. 2017;13(2):152-67.

49. Caccamo A, Majumder S, Richardson A, Strong R, Oddo S. Molecular interplay between mammalian target of rapamycin (mTOR), amyloid-beta, and tau: effects on cognitive impairments. J Biol Chem. 2010;285(17):13107-20.

50. Tramutola A, Triplett JC, Di Domenico F, Niedowicz DM, Murphy MP, Coccia $R$, et al. Alteration of mTOR signaling occurs early in the progression of Alzheimer disease (AD): analysis of brain from subjects with pre-clinical AD, amnestic mild cognitive impairment and late-stage AD. J Neurochem. 2015; 133(5):739-49.

51. Singh AK, Pati U. CHIP stabilizes amyloid precursor protein via proteasomal degradation and p53-mediated trans-repression of beta-secretase. Aging Cell. 2015;14(4):595-604.

52. Jung ES, Choi H, Song H, Hwang YJ, Kim A, Ryu H, et al. p53-dependent SIRT6 expression protects Abeta42-induced DNA damage. Sci Rep. 2016;6:25628.

53. Uberti D, Carsana T, Bernardi E, Rodella L, Grigolato P, Lanni C, et al. Selective impairment of p53-mediated cell death in fibroblasts from sporadic Alzheimer's disease patients. J Cell Sci. 2002;115(Pt 15):3131-8.

54. Uberti D, Lanni C, Carsana T, Francisconi S, Missale C, Racchi M, et al. Identification of a mutant-like conformation of p53 in fibroblasts from sporadic Alzheimer's disease patients. Neurobiol Aging. 2006;27(9):1193-201.

55. Cordani M, Oppici E, Dando I, Butturini E, Dalla Pozza E, Nadal-Serrano M, et al. Mutant p53 proteins counteract autophagic mechanism sensitizing cancer cells to mTOR inhibition. Mol Oncol. 2016;10(7):1008-29.

56. Uberti D, Cenini G, Olivari L, Ferrari-Toninelli G, Porrello E, Cecchi C, et al. Over-expression of amyloid precursor protein in HEK cells alters p53 conformational state and protects against doxorubicin. J Neurochem. 2007; 103(1):322-33.

57. Lanni C, Nardinocchi L, Puca R, Stanga S, Uberti D, Memo M, et al. Homeodomain interacting protein kinase 2: a target for Alzheimer's beta 
amyloid leading to misfolded p53 and inappropriate cell survival. PLoS One. 2010;5(4):e10171.

58. Lanni C, Necchi D, Pinto A, Buoso E, Buizza L, Memo M, et al. Zyxin is a novel target for beta-amyloid peptide: characterization of its role in Alzheimer's pathogenesis. J Neurochem. 2013;125(5):790-9.

59. Buizza L, Cenini G, Lanni C, Ferrari-Toninelli G, Prandelli C, Govoni S, et al. Conformational altered p53 as an early marker of oxidative stress in Alzheimer's disease. PLoS One. 2012;7(1):e29789.

60. Arce-Varas N, Abate G, Prandelli C, Martinez C, Cuetos F, Menendez M, et al. Comparison of extracellular and intracellular blood compartments highlights redox alterations in Alzheimer's and mild cognitive impairment patients. Curr Alzheimer Res. 2017;14(1):112-22.

61. Uberti D, Cenini G, Bonini SA, Barcikowska M, Styczynska M, Szybinska A et al. Increased CD44 gene expression in lymphocytes derived from Alzheimer disease patients. Neurodegener Dis. 2010;7(1-3):143-7.

62. Zhou X, Jia J. P53-mediated G (1)/S checkpoint dysfunction in lymphocytes from Alzheimer's disease patients. Neurosci Lett. 2010;468(3):320-5.

63. Tedeschi A, Nguyen T, Puttagunta R, Gaub P, Di Giovanni S. A p53-CBP/ p300 transcription module is required for GAP-43 expression, axon outgrowth, and regeneration. Cell Death Differ. 2009;16(4):543-54.

64. Buizza L, Prandelli C, Bonini SA, Delbarba A, Cenini G, Lanni C, et al. Conformational altered p53 affects neuronal function: relevance for the response to toxic insult and growth-associated protein 43 expression. Cell Death Dis. 2013;4:e484.

65. de la Monte SM, Ng SC, Hsu DW. Aberrant GAP-43 gene expression in Alzheimer's disease. Am J Pathol. 1995;147(4):934-46.

66. Bogdanovic N, Davidsson P, Volkmann I, Winblad B, Blennow K. Growthassociated protein GAP-43 in the frontal cortex and in the hippocampus in Alzheimer's disease: an immunohistochemical and quantitative study. J Neural Transm (Vienna). 2000;107(4):463-78.

67. Cenini G, Sultana R, Memo M, Butterfield DA. Effects of oxidative and nitrosative stress in brain on $p 53$ proapoptotic protein in amnestic mild cognitive impairment and Alzheimer disease. Free Radic Biol Med. 2008; 45(1):81-5.

68. Farmer KM, Ghag G, Puangmalai N, Montalbano M, Bhatt N, Kayed R. P53 aggregation, interactions with tau, and impaired DNA damage response in Alzheimer's disease. Acta Neuropathol Commun. 2020;8(1):132.

69. Hampel H, O'Bryant SE, Molinuevo JL, Zetterberg H, Masters CL, Lista S, et al. Blood-based biomarkers for Alzheimer disease: mapping the road to the clinic. Nat Rev Neurol. 2018;14(11):639-52.

70. Lanni C, Racchi M, Mazzini G, Ranzenigo A, Polotti R, Sinforiani E, et al. Conformationally altered p53: a novel Alzheimer's disease marker? Mo Psychiatry. 2008;13(6):641-7.

71. Lanni C, Racchi M, Stanga S, Mazzini G, Ranzenigo A, Polotti R, et al. Unfolded p53 in blood as a predictive signature signature of the transition from mild cognitive impairment to Alzheimer's disease. J Alzheimers Dis. 2010;20(1):97-104.

72. Stanga S, Lanni C, Sinforiani E, Mazzini G, Racchi M. Searching for predictive blood biomarkers: misfolded p53 in mild cognitive impairment. Curr Alzheimer Res. 2012:9(10):1191-7.

73. Lanni C, Racchi M, Uberti D, Mazzini G, Stanga S, Sinforiani E, et al. Pharmacogenetics and pharmagenomics, trends in normal and pathological aging studies: focus on p53. Curr Pharm Des. 2008;14(26):2665-71.

74. Memo M, Uberti DL. Antibody binding a linear epitope of human p53 and diagnostic applications thereof. EP3201234; 2018

75. Browne D, McGuinness B, Woodside JV, McKay GJ. Vitamin E and Alzheimer's disease: what do we know so far? Clin Interv Aging. 2019;14 1303-17.

76. Kogan S, Carpizo DR. Zinc metallochaperones as mutant p53 reactivators: a new paradigm in cancer therapeutics. Cancers (Basel). 2018;10(6):166.

\section{Publisher's Note}

Springer Nature remains neutral with regard to jurisdictional claims in published maps and institutional affiliations. 